\title{
Convex duality in optimal investment under illiquidity
}

\author{
Teemu Pennanen*
}

August 16, 2013

\begin{abstract}
We study the problem of optimal investment by embedding it in the general conjugate duality framework of convex analysis. This allows for various extensions to classical models of liquid markets. In particular, we obtain a dual representation for the optimum value function in the presence of portfolio constraints and nonlinear trading costs that are encountered e.g. in modern limit order markets. The optimization problem is parameterized by a sequence of financial claims. Such a parameterization is essential in markets without a numeraire asset when pricing swap contracts and other financial products with multiple payout dates. In the special case of perfectly liquid markets or markets with proportional transaction costs, we recover well-known dual expressions in terms of martingale measures.
\end{abstract}

Key words: Optimal investment, illiquidity, convex duality

\section{Introduction}

Convex duality has long been an integral part of mathematical finance. Classical references include Harrison and Kreps [15], Harrison and Pliska [16], Kreps [25] and Dalang et al. [8] where the no-arbitrage principle behind the Black-Scholes formula was related to the existence of certain dual variables; see Delbaen and Schachermayer [10] for a detailed discussion of the topic. In problems of optimal investment, convex duality has become an important tool in the analysis of optimal solutions; see e.g. Kramkov and Schachermayer [23], Delbaen et al. [9], Karatzas and Žitković [21] and Rogers [40] and their references.

This paper studies convex duality in problems of optimal investment in illiquid financial markets where one may encounter frictions or restrictions when transferring wealth through time or between assets. Our model extends the classical linear model by allowing for portfolio constraints and nonlinear transaction costs that are encountered e.g. in limit order markets. In particular, we

*Department of Mathematics, King's College London, teemu.pennanen@kcl.ac.uk 
do not assume, a priori, the existence of a numeraire that allows for free transfer of (both positive as well as negative amounts of) wealth through time. This is a significant departure from the classical model where a numeraire is assumed in order to express wealth processes in terms of stochastic integrals. Such models are at odds with real markets where much of trading consists of exchanging sequences of cash-flows. Such trades would be unnecessary if one could postpone payments to a single date by shorting the numeraire.

While in classical models of perfectly liquid markets, convex analysis can often be reduced to an application of basic separation theorem, in the presence of nonlinear illiquidity effects, more sophisticated convex analysis is needed; see for example Cvitanić and Karatzas [6], Jouini and Kallal [18, 17], Guasoni [14], Schachermayer [43], Rokhlin [41], Bielecki, Cialenco and Rodriguez [4] as well as the books of Kabanov and Safarian [19] and Rogers [40]. The present paper builds on the market model introduced in Pennanen [30] where trading costs and portfolio constraints are described by general convex normal integrands and measurable closed convex sets, respectively. This discrete-time model provides a unifying framework for modeling transaction costs and portfolio constraints as well as nonlinear illiquidity effects.

The classical superhedging principle was extended to this model in [33, 32]. The present paper makes similar extensions to the duality theory of optimal investment. More precisely, we derive a dual representation for the optimal value function of the optimal investment problem studied in [34] as a basis for valuation of financial contracts. The dual representation is obtained by applying the stochastic optimization duality framework developed in [31] as an instance of the conjugate duality framework of Rockafellar [38]. This allows for a unified treatment of many well known models of mathematical finance where dual correspondences have often been derived case by case. In particular, we illustrate how the dual variables in the general case are connected to martingale measures and shadow prices that have been extensively studied in models with a numeraire.

\section{Optimal investment}

This section recalls the optimal investment problem from [34]. The problem is parameterized by financial liabilities characterized by a sequence of cash-flows the agent has to deliver over time. The dependence of the optimum value on the liabilities is important in valuation of swap contracts and other financial products that provide payments at multiple points in time. Section 3 derives dual expressions for the optimum value function.

Consider a financial market where a finite set $J$ of assets can be traded over finite discrete time $t=0, \ldots, T$. We will model uncertainty by a probability space $(\Omega, \mathcal{F}, P)$ and the information by a nondecreasing sequence $\left(\mathcal{F}_{t}\right)_{t=0}^{T}$ of sub-sigma algebras of $\mathcal{F}$. At time $t$ one does not know which scenario $\omega \in \Omega$ will realize but only to which element of $\mathcal{F}_{t}$ it belongs to. The filtration property $\mathcal{F}_{t} \subseteq \mathcal{F}_{t+1}$ means that information increases over time. 
We describe trading strategies by $\left(\mathcal{F}_{t}\right)_{t=0}^{T}$-adapted sequences $x=\left(x_{t}\right)_{t=0}^{T}$ of $\mathbb{R}^{J}$-valued stochastic processes. The random vector $x_{t}$ describes the portfolio of assets held over $(t, t+1]$. The adaptedness means that $x_{t}$ is $\mathcal{F}_{t}$-measurable, i.e. the portfolio chosen at time $t$ only depends on information available at time $t$. The linear space of adapted trading strategies will be denoted by $\mathcal{N}$. Unless $\mathcal{F}_{T}$ has only a finite number of elements with positive probability, $\mathcal{N}$ is an infinite-dimensional space. We will assume that $\mathcal{F}_{0}=\{\Omega, \emptyset\}$ so that $x_{0}$ is independent of $\omega$. Implementing a portfolio process $x \in \mathcal{N}$ requires buying a portfolio $\Delta x_{t}:=x_{t}-x_{t-1}$ of assets at time $t$. Negative purchases are interpreted as sales.

Trading costs will be described by an $\left(\mathcal{F}_{t}\right)_{t=0^{-}}^{T}$ adapted sequence $S=\left(S_{t}\right)_{t=0}^{T}$ of convex normal integrands on $\mathbb{R}^{J} \times \mathcal{F}$. This means that for each $t$, the setvalued mapping $\omega \mapsto\left\{(x, \alpha) \in \mathbb{R}^{J} \times \mathbb{R} \mid S_{t}(x, \omega) \leq \alpha\right\}$ is closed convex-valued and $\mathcal{F}_{t}$-measurable ${ }^{1}$. The value of $S_{t}(x, \omega)$ is interpreted as the cost of buying a portfolio $x \in \mathbb{R}^{J}$ at time $t$ in state $\omega$. Accordingly, we assume that $S_{t}(0, \omega)=0$. Implementing a trading strategy $x \in \mathcal{N}$ requires investing $S_{t}\left(\Delta x_{t}\right)$ units of cash at time $t$. The measurability condition on $S_{t}$ implies that $\omega \mapsto S_{t}\left(\Delta x_{t}(\omega), \omega\right)$ is $\mathcal{F}_{t}$-measurable, i.e. the cost of a portfolio is known at the time of purchase. Indeed, the cost is the composition of the measurable functions $\omega \mapsto\left(\Delta x_{t}(\omega), \omega\right)$ and $S_{t}$; see e.g. [39, Corollary 14.34].

The classical model of perfectly liquid markets corresponds to $S_{t}(x, \omega)=$ $s_{t}(\omega) \cdot x$ where $s=\left(s_{t}\right)_{t=0}$ is an $\left(\mathcal{F}_{t}\right)_{t=0}^{T}$-adapted sequence of price vectors independent of the traded amounts. Markets with proportional transaction costs and/or bid-ask-spreads can be modeled with sublinear cost functions

$$
S_{t}(x, \omega)=\sup \left\{s \cdot x \mid s \in\left[s_{t}^{-}(\omega), s_{t}^{+}(\omega)\right]\right\},
$$

where the $\left(\mathcal{F}_{t}\right)_{t=0}^{T}$-adapted $\mathbb{R}^{J}$-valued processes $s^{-}$and $s^{+}$give the bid- and ask-prices, respectively; see [18]. Convex trading costs arise naturally also in modern limit order markets, where the cost of a "market order" is nonlinear and convex in the traded amount. Parametric convex market models have been proposed e.g. in Cetin and Rogers [5] and Malo and Pennanen [26].

We model portfolio constraints by requiring that the portfolio $x_{t}$ chosen at time $t$ has to belong to a closed convex set $D_{t}$ much as e.g. in $[28,41,11]$. We allow $D_{t}$ to be random but assume that it is $\mathcal{F}_{t}$-measurable. We assume that $0 \in D_{t}$ almost surely, i.e. that the zero portfolio is feasible. The classical unconstrained model corresponds to $D \equiv \mathbb{R}^{J}$ while short selling constraints, studied e.g. in Cvitanic and Karatzas [6] and Jouini and Kallal [17] can be described by $D \equiv \mathbb{R}_{+}^{J}$. As observed there, short selling constraints can also be used to model different interest rates for lending and borrowing. Indeed, this can be done by introducing lending and borrowing accounts whose unit prices appreciate at lending and borrowing rates, respectively, and by restricting the investments in these assets to be nonnegative and nonpositive, respectively.

\footnotetext{
${ }^{1}$ A set-valued mapping $T: \Omega \rightrightarrows \mathbb{R}^{n}$ is $\mathcal{F}_{t}$-measurable if $\{\omega \in \Omega \mid T(\omega) \cap U \neq \emptyset\} \in \mathcal{F}_{t}$ for every open $U \subset \mathbb{R}^{n}$.
} 
Consider now an agent whose financial position is described by an $\left(\mathcal{F}_{t}\right)_{t=0^{-}}^{T}$ adapted sequence of cash-flows $c=\left(c_{t}\right)_{t=0}^{T}$ in the sense that the agent has to deliver a random amount $c_{t}$ of cash at time $t$. We allow $c_{t}$ to take both positive and negative values so it may describe both endowments as well as liabilities. In particular, $-c_{0}$ may be interpreted as an initial endowment while the subsequent payments $c_{t}, t=1, \ldots, T$ may be interpreted as the cash-flows associated with financial liabilities. We will denote the linear space of $\left(\mathcal{F}_{t}\right)_{t=0}^{T}$-adapted sequences of cash-flows by

$$
\mathcal{M}=\left\{\left(c_{t}\right)_{t=0}^{T} \mid c_{t} \in L^{0}\left(\Omega, \mathcal{F}_{t}, P\right)\right\} .
$$

Here and in what follows, $L^{0}\left(\Omega, \mathcal{F}_{t}, P\right)$ stands for the linear space of equivalence classes of $\mathcal{F}_{t}$-measurable real-valued functions. As usual, two measurable functions are equivalent if they coincide $P$-almost surely. Given $c \in \mathcal{M}$, and nondecreasing ${ }^{2}$ convex functions $\mathcal{V}_{t}$ on $L^{0}\left(\Omega, \mathcal{F}_{t}, P\right)$, we will study the problem

$$
\text { minimize } \quad \sum_{t=0}^{T} \mathcal{V}_{t}\left(S_{t}\left(\Delta x_{t}\right)+c_{t}\right) \quad \text { over } \quad x \in \mathcal{N}_{D},
$$

where $\mathcal{N}_{D}:=\left\{x \in \mathcal{N} \mid x_{t} \in D_{t}, t=0, \ldots, T-1, x_{T}=0\right\}$ and $\Delta x_{t}:=x_{t}-x_{t-1}$. We always define $x_{-1}=0$ so the elements of $\mathcal{N}_{D}$ describe trading strategies that start and end at liquidated positions. The functions $\mathcal{V}_{t}$ measure the disutility (regret, loss, ...) caused by the net expenditure $S_{t}\left(\Delta x_{t}\right)+c_{t}$ of updating the portfolio and paying out the claim $c_{t}$ at time $t$. We allow $\mathcal{V}_{t}$ to be extended realvalued but assume that $\mathcal{V}_{t}(0)=0$. We interpret the value of $\mathcal{V}_{t}\left(S_{t}\left(\Delta x_{t}\right)+c_{t}\right)$ as $+\infty$ unless $S_{t}\left(\Delta x_{t}\right) \in L^{0}\left(\Omega, \mathcal{F}_{t}, P\right)$. In other words, the formulation of (ALM) includes the implicit constraint that $\Delta x_{t} \in \operatorname{dom} S_{t}$ almost surely.

Problem (ALM) can be interpreted as an asset-liability management problem where one looks for a trading strategy whose proceeds fit the liabilities $c \in \mathcal{M}$ optimally as measured by the "disutility functions" $\mathcal{V}_{t}$. Despite the simple appearance, (ALM) covers many more familiar instances of portfolio optimization problems. In particular, when ${ }^{3} \mathcal{V}_{t}=\delta_{L_{-}^{0}}$ for $t<T$, we can write it as

$$
\begin{array}{ll}
\operatorname{minimize} & \mathcal{V}_{T}\left(S_{T}\left(\Delta x_{T}\right)+c_{T}\right) \quad \text { over } \quad x \in \mathcal{N}_{D} \\
\text { subject to } & S_{t}\left(\Delta x_{t}\right)+c_{t} \leq 0, \quad t=0, \ldots, T-1
\end{array}
$$

This is an illiquid version of the classical utility maximization problem.

Example 1 (Numeraire and stochastic integration) Assume, as e.g. in Çetin and Rogers [5] and Czichowsky et al. [7], that there is a perfectly liquid asset (numeraire), say $0 \in J$, such that

$$
S_{t}(x)=x^{0}+\tilde{S}_{t}(\tilde{x}) \quad \text { and } \quad D_{t}=\mathbb{R} \times \tilde{D}_{t},
$$

\footnotetext{
${ }^{2} \mathrm{~A}$ function $\mathcal{V}_{t}: L^{0}\left(\Omega, \mathcal{F}_{t}, P\right) \rightarrow \overline{\mathbb{R}}$ is nondecreasing if $\mathcal{V}_{t}\left(c_{t}^{1}\right) \leq \mathcal{V}_{t}\left(c_{t}^{2}\right)$ whenever $c_{t}^{1} \leq c_{t}^{2}$ almost surely.

${ }^{3}$ Here and in what follows, $\delta_{C}$ denotes the indicator function of a set $C: \delta_{C}(x)$ equals 0 or $+\infty$ depending on whether $x \in C$ or not.
} 
where $\tilde{S}$ and $\tilde{D}$ are the cost process and the constraints for the remaining risky assets $\tilde{J}=J \backslash\{0\}$. We can then use the budget constraint in (1) to substitute out the numeraire from the problem. Indeed, defining

$$
x_{t}^{0}=x_{t-1}^{0}-\tilde{S}_{t}\left(\Delta \tilde{x}_{t}\right)-c_{t} \quad t=0, \ldots, T-1,
$$

the budget constraint holds as an equality for $t=1, \ldots, T-1$ and

$$
x_{T-1}^{0}=-\sum_{t=0}^{T-1} \tilde{S}_{t}\left(\Delta \tilde{x}_{t}\right)-\sum_{t=0}^{T-1} c_{t} .
$$

Substituting this in the objective (and recalling that $x_{T}=0$ for $x \in \mathcal{N}_{D}$ ), problem (1) becomes

$$
\text { minimize } \mathcal{V}_{T}\left(\sum_{t=0}^{T} \tilde{S}_{t}\left(\Delta \tilde{x}_{t}\right)+\sum_{t=0}^{T} c_{t}\right) \quad \text { over } \quad x \in \mathcal{N}_{D},
$$

which shows that in the presence of a numeraire, the timing of payments is irrelevant. This is the problem studied in [5] in the case of strictly convex $\tilde{S}_{t}$ and in [7] in the case of sublinear $\tilde{S}_{t}$. In linear market models with $\tilde{S}_{t}(\tilde{x}, \omega)=$ $\tilde{s}_{t}(\omega) \cdot \tilde{x}_{t}$, we can express the accumulated trading costs as a stochastic integral,

$$
\sum_{t=0}^{T} \tilde{S}_{t}\left(\Delta \tilde{x}_{t}\right)=\sum_{t=0}^{T} \tilde{s}_{t} \cdot \Delta \tilde{x}_{t}=-\sum_{t=0}^{T-1} \tilde{x}_{t} \cdot \Delta \tilde{s}_{t+1} .
$$

We then recover constrained discrete-time versions of the utility maximization problems studied e.g. in [23, 9, 24, 2, 3]. In [23, 24, 2], the financial position of the agent was described solely in terms of an initial endowment $w \in \mathbb{R}$ without future liabilities. This corresponds to $c_{0}=-w$ and $c_{t}=0$ for $t>0$.

We will denote the optimal value of problem (ALM) by

$$
\varphi(c):=\inf _{x \in \mathcal{N}_{D}} \sum_{t=0}^{T} \mathcal{V}_{t}\left(S_{t}\left(\Delta x_{t}\right)+c_{t}\right) .
$$

It is easily verified that $\varphi$ is a convex function on $\mathcal{M}$. The value function $\varphi$ has a central role in the valuation of financial contracts with multiple payout dates; see [34]. In the completely risk averse case where $\mathcal{V}_{t}=\delta_{L_{-}^{0}}$ for all $t=0, \ldots, T$, we get $\varphi=\delta_{\mathcal{C}}$, where

$$
\mathcal{C}=\left\{c \in \mathcal{M} \mid \exists x \in \mathcal{N}_{D}: S_{t}\left(\Delta x_{t}\right)+c_{t} \leq 0 \quad \forall t\right\}
$$

is the set of claim processes that can be superhedged without a cost; see [33] for further discussion and references on superhedging. On the other hand, since the functions $\mathcal{V}_{t}$ are nondecreasing, we can write $\varphi$ as the infimal convolution

$$
\begin{aligned}
\varphi(c) & =\inf _{d \in \mathcal{M}}\left\{\sum_{t=0}^{T} \mathcal{V}_{t}\left(d_{t}\right) \mid c-d \in \mathcal{C}\right\} \\
& =\inf _{d \in \mathcal{C}} \sum_{t=0}^{T} \mathcal{V}_{t}\left(c_{t}-d_{t}\right),
\end{aligned}
$$


of $\delta_{\mathcal{C}}$ and the function

$$
\mathcal{V}(d)=\sum_{t=0}^{T} \mathcal{V}_{t}\left(d_{t}\right)
$$

as is seen by first writing (ALM) in the form

$$
\begin{array}{ll}
\text { minimize } & \sum_{t=0}^{T} \mathcal{V}_{t}\left(d_{t}\right) \quad \text { over } \quad x \in \mathcal{N}, d \in \mathcal{M} \\
\text { subject to } & S_{t}\left(\Delta x_{t}\right)+c_{t} \leq d_{t}, \quad x_{t} \in D_{t} .
\end{array}
$$

The variable $d$ may be interpreted as investments the agent makes to his portfolio over time. Alternatively, one may interpret $-c$ and $-d$ as endowment and consumption, respectively, in the spirit of the optimal consumption-investment problem of Karatzas and Zitković [21] in liquid markets in continuous time.

\section{Duality}

Prices of financial products are often expressed in terms of dual variables of one kind or another. Prices of bonds can be expressed in terms of zero curves, which represent the time-value of money. In models with a cash-account, on the other hand, prices of random cash-flows are often expressed in terms of martingale measures. In illiquid markets without a cash-account, one needs more general dual variables that encompass both the time value of money as well as the randomness. This section derives a dual representation for the value function $\varphi$ of (ALM) in terms of such variables. When specialized to more traditional market models, we recover (discrete-time versions of) some well-known duality results for portfolio optimization. Pricing formulas for contingent claims can then be derived from the general relationships between the optimal investment and contingent claim valuation; see [34].

Our strategy is to embed (ALM) in the general stochastic optimization duality framework of [31] which is essentially an instance of the classical conjugate duality framework of Rockafellar [38]. The bilinear form

$$
\langle c, y\rangle:=E \sum_{t=0}^{T} c_{t} y_{t}
$$

puts the space

$$
\mathcal{M}^{1}:=\left\{\left(c_{t}\right)_{t=0}^{T} \mid c_{t} \in L^{1}\left(\Omega, \mathcal{F}_{t}, P\right)\right\}
$$

of integrable sequences of cash-flows in separating duality with the space

$$
\mathcal{M}^{\infty}:=\left\{\left(y_{t}\right)_{t=0}^{T} \mid y_{t} \in L^{\infty}\left(\Omega, \mathcal{F}_{t}, P\right)\right\}
$$

of essentially bounded adapted processes. Given a convex function $f$ on $\mathcal{M}^{1}$, its conjugate on $\mathcal{M}^{\infty}$ is defined by

$$
f^{*}(y)=\sup _{c \in \mathcal{M}^{1}}\{\langle c, y\rangle-f(c)\} .
$$


Being the pointwise supremum of continuous linear functions, $f^{*}$ is convex and lower semicontinuous with respect to the weak topology $\sigma\left(\mathcal{M}^{\infty}, \mathcal{M}^{1}\right)$. The classical biconjugate theorem says that if $f$ is proper and lower semicontinuous with respect to the $L^{1}$-norm, then it has the dual representation

$$
f(c)=\sup _{y \in \mathcal{M}^{\infty}}\left\{\langle c, y\rangle-f^{*}(y)\right\}
$$

see Moreau [27]. This abstract formula is behind many fundamental duality results in mathematical finance. In order to apply it to (ALM), we will first establish the lower semicontinuity of the value function $\varphi$.

We will assume from now on that

$$
\mathcal{V}_{t}\left(c_{t}\right)=E v_{t}\left(c_{t}\right):=\int v_{t}\left(c_{t}(\omega), \omega\right) d P(\omega),
$$

where $v_{t}$ is an $\mathcal{F}_{t}$-measurable normal integrand on $\mathbb{R} \times \Omega$ such that $v_{t}(\cdot, \omega)$ is proper, nondecreasing and convex with $v_{t}(0, \omega)=0$ for every $\omega \in \Omega$. As usual, we define the integral of a measurable function as $+\infty$ unless the positive part of the function is integrable.

Given a market model $(S, D)$, we obtain another market model $\left(S^{\infty}, D^{\infty}\right)$ by defining $S_{t}^{\infty}(\cdot, \omega)$ and $D_{t}^{\infty}(\omega)$ pointwise as the recession function and recession cone of $S_{t}(\cdot, \omega)$ and $D_{t}(\omega)$, respectively. When $S$ is sublinear and $D$ is conical, we simply have $(S, D)=\left(S^{\infty}, D^{\infty}\right)$. By [37, Corollary 8.3.2 and Theorem 8.5],

$$
\begin{aligned}
S_{t}^{\infty}(x, \omega) & =\sup _{\alpha>0} \frac{S_{t}(\alpha x, \omega)}{\alpha}, \\
D_{t}^{\infty}(\omega) & =\bigcap_{\alpha>0} \alpha D_{t}(\omega) .
\end{aligned}
$$

The required measurability properties hold by [39, Exercises 14.54 and 14.21] while the convexity and topological properties come directly from the definitions. The following is derived in [34] from a more general result of [35] on stochastic optimization.

Theorem 2 Assume that $\left\{x \in \mathcal{N}_{D^{\infty}} \mid S_{t}^{\infty}\left(\Delta x_{t}\right) \leq 0\right\}$ is a linear space and that $v_{t}(\cdot, \omega)$ are nonconstant functions with $v_{t} \geq m$ for some integrable function $m \in L^{1}$. Then the value function $\varphi$ is a proper lower semicontinuous convex function on $\mathcal{M}^{1}$ and the infimum in (ALM) is attained for every $c \in \mathcal{M}^{1}$.

The linearity condition in Theorem 2 is a generalization of the no-arbitrage condition in classical perfectly liquid markets; see [32, Section 4]. In particular, when $S_{t}(x)=s_{t} \cdot x$ and $D_{t} \equiv \mathbb{R}^{J}$, the linearity condition means that any $x \in \mathcal{N}_{D}$ with $s_{t} \cdot \Delta x \leq 0$ almost surely for all $t$ has $s_{t} \cdot \Delta x=0$ almost surely for all $t$, i.e. there are no self-financing trading strategies that generate nonzero revenue. This is exactly the no-arbitrage condition. In nonlinear market models, the linearity condition is implied by the so called "robust no-arbitrage" condition; see [32, Section 4]. In the most risk averse case where $v_{t}=\delta_{\mathbb{R}_{-}}$for every $t$, Theorem 2 
says that the set $\mathcal{C}$ is closed. In the classical linear model of Example 1, we thus recover the key closedness result of Schachermayer [42, Lemma 2.1]. The linearity condition holds also if $D_{t}^{\infty}(\omega) \cap\left\{x \in \mathbb{R}^{J} \mid S_{t}^{\infty}(x, \omega) \leq 0\right\}=\{0\}$ almost surely for every $t$. Indeed, since $x_{-1}=0$, by definition, this condition implies $\left\{x \in \mathcal{N}_{D^{\infty}} \mid S_{t}^{\infty}\left(\Delta x_{t}\right) \leq 0\right\}=\{0\}$. This certainly holds if $D_{t}^{\infty} \subseteq \mathbb{R}_{+}^{J}$ and $\left\{x \in \mathbb{R}^{J} \mid S_{t}^{\infty}(x, \omega) \leq 0\right\} \cap \mathbb{R}_{+}^{J}=\{0\}$. Here, the first condition means that infinite short positions are prohibited while the second means that there are no completely worthless assets.

Nonconstancy of $v_{t}(\cdot, \omega)$ in Theorem 2 simply means that the investor always prefers more money to less. The lower bound is a more significant restriction since it excludes e.g. the logarithmic utility. In Kramkov and Schachermayer [23] and Rasonyi and Stettner [36] such bounds were avoided but, at present, it is unclear if the lower bound can be relaxed in illiquid markets. However, Guasoni [13, Theorem 5.2] gives the existence of optimal solutions in a continuous time model with a cash-account and proportional transaction with similar conditions on the utility function as in [23] and [36]. Much like in [36] and the proof of Theorem 2, his approach was based on the "direct method" rather than duality arguments as e.g. in [23].

While Theorem 2 establishes the validity of the dual representation for the value function $\varphi$, its conjugate $\varphi^{*}$ can be expressed in terms of the support function

$$
\sigma_{\mathcal{C}}(y)=\sup _{c \in \mathcal{M}^{1}}\{\langle c, y\rangle \mid c \in \mathcal{C}\}
$$

of $\mathcal{C}$ and the conjugates

$$
v_{t}^{*}(y, \omega)=\sup _{c \in \mathbb{R}}\left\{c y-v_{t}(c, \omega)\right\}
$$

of the disutility functions $v_{t}$ as follows.

Lemma 3 The conjugate of $\varphi$ can be expressed as

$$
\varphi^{*}(y)=\sigma_{\mathcal{C}}(y)+E \sum_{t=0}^{T} v_{t}^{*}\left(y_{t}\right)
$$

Proof. Problem (ALM) can be written as

$$
\text { minimize } E f(x, d, c) \quad \text { over } \quad x \in \mathcal{N}, d \in \mathcal{M}
$$

where $f:\left(\mathbb{R}^{J}\right)^{T+1} \times \mathbb{R}^{T+1} \times \mathbb{R}^{T+1} \times \Omega \rightarrow \overline{\mathbb{R}}$ is defined

$$
f(x, d, c, \omega)= \begin{cases}\sum_{t=0}^{T} v_{t}\left(d_{t}, \omega\right) & \text { if } S_{t}\left(\Delta x_{t}, \omega\right)+c_{t} \leq d_{t}, x_{t} \in D_{t}(\omega), x_{T}=0 \\ +\infty & \text { otherwise }\end{cases}
$$

Since $v_{t}$ and $S_{t}$ are normal integrands and $D_{t}$ are measurable, it follows from Theorem 14.36 and Proposition 14.44 of [39] that $f$ is a normal integrand. We 
are thus in the general stochastic optimization framework of [31] so, by [31, Theorem 2.2],

$$
-\varphi^{*}(y)=\inf _{x \in \mathcal{N}, d \in \mathcal{M}} E l(x, d, y)
$$

where $l(x, d, y, \omega)=\inf _{c \in \mathbb{R}^{T+1}}\left\{f(x, d, c, \omega)-\sum_{t=0}^{T} c_{t} y_{t}\right\}$. We can write $l$ as

$$
l(x, d, y, \omega)= \begin{cases}+\infty & \text { unless } x \in X(\omega), \\ \sum_{t=0}^{T}\left[v_{t}\left(d_{t}, \omega\right)+y_{t} S_{t}\left(\Delta x_{t}, \omega\right)-d_{t} y_{t}\right] & \text { if } x \in X(\omega) \text { and } y \geq 0 \\ -\infty & \text { otherwise }\end{cases}
$$

where $X(\omega)=\left\{x \in\left(\mathbb{R}^{J}\right)^{T+1} \mid \Delta x_{t} \in \operatorname{dom} S_{t}(\cdot, \omega), x_{t} \in D_{t}(\omega) \forall t\right\}$. Unless $y \geq 0$ almost surely, the infimum in (5) equals $-\infty$ (it is attained e.g. by $(x, d)=(0,0))$. For $y \geq 0$,

$$
\begin{aligned}
-\varphi^{*}(y) & =\inf _{x \in \mathcal{N}, d \in \mathcal{M}}\left\{E \sum_{t=0}^{T}\left[y_{t} S_{t}\left(\Delta x_{t}\right)+v_{t}\left(d_{t}\right)-d_{t} y_{t}\right] \mid x \in X\right\} \\
& =\inf _{x \in \mathcal{N}}\left\{E \sum_{t=0}^{T} y_{t} S_{t}\left(\Delta x_{t}\right) \mid x \in X\right\}+\inf _{d \in \mathcal{M}} E \sum_{t=0}^{T}\left[v_{t}\left(d_{t}\right)-d_{t} y_{t}\right] \\
& =\inf _{x \in \mathcal{N}}\left\{E \sum_{t=0}^{T} y_{t} S_{t}\left(\Delta x_{t}\right) \mid x \in X\right\}-E \sum_{t=0}^{T} v_{t}^{*}\left(y_{t}\right),
\end{aligned}
$$

where the second equality holds since we can restrict the minimization, without affecting the infimum, to those $x \in \mathcal{N}$ and $d \in \mathcal{M}$ for which both integrands have integrable positive parts. The last equality comes from the interchange rule for normal integrands; see e.g. [39, Theorem 14.60]. A similar argument in the case $v_{t} \equiv \delta_{\mathbb{R}_{-}}$, shows that

$$
-\sigma_{\mathcal{C}}(y)=\inf _{x \in \mathcal{N}}\left\{E \sum_{t=0}^{T} y_{t} S_{t}\left(\Delta x_{t}\right) \mid x \in X\right\}
$$

for $y \geq 0$ while $\sigma_{\mathcal{C}}(y)=\infty$ for $y \nsupseteq 0$.

The form of the conjugate in Lemma 3 is not surprising given the expression (2) of $\varphi$ as the infimal convolution of $\delta_{\mathcal{C}}$ and $\mathcal{V}$. However, the infimal convolution is taken in the space $\mathcal{M}$ of all adapted claim processes, not over $\mathcal{M}^{1}$. One could, of course, concentrate on integrable claim processes from the beginning and study the function

$$
\tilde{\varphi}(c)=\inf _{d \in \mathcal{M}^{1}}\left\{E \sum_{t=0}^{T} v_{t}\left(d_{t}\right) \mid c-d \in \mathcal{C}\right\}
$$

which has the same conjugate and essentially the same economic interpretation as $\varphi$. However, the proof of Theorem 2 breaks down if $d \in \mathcal{M}$ is restricted to $\mathcal{M}^{1}$ 
and we do not know whether $\tilde{\varphi}$ is lower semicontinuous under the assumptions of Theorem 2.

Combining Theorem 2 and Lemma 3 with the biconjugate theorem gives the following.

Theorem 4 Under assumptions of Theorem 2, the value function of (ALM) has the dual representation

$$
\varphi(c)=\sup _{y \in \mathcal{M}^{\infty}}\left\{\langle c, y\rangle-\sigma_{\mathcal{C}}(y)-E \sum_{t=0}^{T} v_{t}^{*}\left(y_{t}\right)\right\} .
$$

In general, the supremum in the above expression need not be attained; see Remark 7 below. When $\mathcal{C}$ is a cone, the dual representation can be written

$$
\varphi(c)=\sup _{y \in \mathcal{C}^{*}}\left\{\langle c, y\rangle-E \sum_{t=0}^{T} v_{t}^{*}\left(y_{t}\right)\right\}
$$

where $\mathcal{C}^{*}:=\left\{y \in \mathcal{M}^{\infty} \mid\langle c, y\rangle \leq 0 \forall c \in \mathcal{C} \cap \mathcal{M}^{1}\right\}$ is the polar cone of $\mathcal{C}$. This is similar in form with the primal formulation

$$
\varphi(c)=\inf _{d \in \mathcal{C}}\left\{E \sum_{t=0}^{T} v_{t}\left(c_{t}-d_{t}\right)\right\} .
$$

Example 5 Much of duality theory in optimal investment has studied the optimum value as a function of the initial endowment only; see e.g. Kramkov and Schachermayer [23] or Klein and Rogers [22]. The function $V\left(c_{0}\right)=$ $\varphi\left(c_{0}, 0, \ldots, 0\right)$ gives the optimum value of (ALM) for an agent with initial capital $-c_{0}$ and no future liabilities/endowments. Using (2), we can write this as

$$
V\left(c_{0}\right)=\inf _{d \in \mathcal{C}\left(c_{0}\right)} E \sum_{t=0}^{T} v_{t}\left(d_{t}\right)
$$

where the set $\mathcal{C}\left(c_{0}\right)=\left\{d \in \mathcal{M} \mid\left(c_{0}, 0, \ldots, 0\right)-d \in \mathcal{C}\right\}$ consists of sequences of investments needed to finance a riskless trading strategy when starting with initial capital $-c_{0}$. If $\varphi$ is proper and lower semicontinuous (see Theorem 2), the biconjugate theorem gives

$$
V\left(c_{0}\right)=\sup _{y \in \mathcal{M}^{\infty}}\left\{c_{0} y_{0}-\varphi^{*}(y)\right\}=\sup _{y_{0} \in \mathbb{R}}\left\{c_{0} y_{0}-U\left(y_{0}\right)\right\}
$$

where

$$
U\left(y_{0}\right)=\inf _{z \in \mathcal{M}^{\infty}}\left\{\varphi^{*}(z) \mid z_{0}=y_{0}\right\} .
$$

If $\mathcal{C}$ is conical, we can use Lemma 3 to write $U$ analogously to (6) as

$$
U\left(y_{0}\right)=\inf _{z \in \mathcal{Y}\left(y_{0}\right)} E \sum_{t=0}^{T} v_{t}^{*}\left(z_{t}\right),
$$


where $\mathcal{Y}\left(y_{0}\right)=\left\{z \in \mathcal{C}^{*} \mid z_{0}=y_{0}\right\}$. In general, there is no reason to believe that $U$ is lower semicontinuous nor that the infimum in its definition is attained. In some cases, however, it is possible to enlarge the set $\mathcal{Y}\left(y_{0}\right)$ so that the function $U$ becomes lower semicontinuous and the infimum is attained; see [7, Section 4] for details.

In order to relate the above to more familiar duality results in optimal investment, we need an explicit expression for the support function $\sigma_{\mathcal{C}}$. A cost process $S$ is said to be integrable if $S_{t}(x, \cdot)$ is integrable for every $x \in \mathbb{R}^{J}$ and $t=0, \ldots, T$. Integrability implies that $\operatorname{dom} S_{t}(\cdot, \omega)=\mathbb{R}^{J}$ almost surely ${ }^{4}$. A linear cost process $S_{t}(x, \omega)=s_{t}(\omega) \cdot x$ is integrable if and only if $s_{t}$ are integrable. The following result, where $\mathcal{N}^{1}$ denotes the space of integrable $\mathbb{R}^{J}$-valued adapted processes and $E_{t}$ the conditional expectation with respect to $\mathcal{F}_{t}$, is from [30, Lemma A1]. Its proof is based on the classical Fenchel-Moreau-Rockafellar duality theorem.

Lemma 6 If $S$ is integrable, then

$$
\sigma_{\mathcal{C}}(y)=\inf _{z \in \mathcal{N}^{1}}\left\{\sum_{t=0}^{T} E\left(y_{t} S_{t}\right)^{*}\left(z_{t}\right)+\sum_{t=0}^{T-1} E \sigma_{D_{t}}\left(E_{t} \Delta z_{t+1}\right)\right\}
$$

for every $y \in \mathcal{M}_{+}^{\infty}$ while $\sigma_{\mathcal{C}}(y)=+\infty$ for $y \notin \mathcal{M}_{+}^{\infty}$. Moreover, the infimum is attained for every $y \in \mathcal{M}_{+}^{\infty}$.

If $S$ is sublinear and integrable (so that $\operatorname{dom} S_{t}=\mathbb{R}^{J}$ and $S_{t}^{*}$ is the indicator of its domain), we get $\left(y_{t} S_{t}\right)^{*}(z)=\delta\left(z \mid y_{t} \operatorname{dom} S_{t}^{*}\right)$. If in addition, $D$ is conical, we have $\sigma_{D_{t}}=\delta_{D_{t}^{*}}$ and

$$
\sigma_{\mathcal{C}}=\delta_{\mathcal{C}^{*}}
$$

where, by Lemma 6 ,

$$
\mathcal{C}^{*}=\left\{y \in \mathcal{M}_{+}^{\infty} \mid \exists z \in \mathcal{N}^{1}: z_{t} \in y_{t} \operatorname{dom} S_{t}^{*}, E_{t}\left[\Delta z_{t+1}\right] \in D_{t}^{*}\right\} .
$$

The polar cone of $\mathcal{C}$ can also be written as ${ }^{5}$

$$
\mathcal{C}^{*}=\left\{y \in \mathcal{M}_{+}^{\infty} \mid \exists s \in \mathcal{N}: s_{t} \in \operatorname{dom} S_{t}^{*}, E_{t}\left[\Delta\left(y_{t+1} s_{t+1}\right)\right] \in D_{t}^{*}\right\} .
$$

In unconstrained linear models with $S_{t}(x, \omega)=s_{t}(\omega) \cdot x$ and $D_{t}(\omega)=\mathbb{R}^{J}$, we get $\operatorname{dom} S_{t}^{*}=\left\{s_{t}\right\}$ and $D_{t}^{*}(\omega)=\{0\}$ so that

$$
\mathcal{C}^{*}=\left\{y \in \mathcal{M}_{+}^{\infty} \mid y s \text { is a martingale }\right\},
$$

while in models with bid-ask spreads, we get $\operatorname{dom} S_{t}^{*}=\left[s_{t}^{-}, s_{t}^{+}\right]$and

$$
\mathcal{C}^{*}=\left\{y \in \mathcal{M}_{+}^{\infty} \mid \exists s \in \mathcal{N}: s_{t}^{-} \leq s_{t} \leq s_{t}^{+}, y s \text { is a martingale }\right\} .
$$

When short selling is prohibited, i.e. when $D_{t}=\mathbb{R}_{+}^{J}$, the condition $E_{t}\left[\Delta\left(y_{t+1} s_{t+1}\right)\right] \in$ $D_{t}^{*}$ means that $y s$ is a supermartingale.

\footnotetext{
${ }^{4}$ Integrability implies $P\left(S_{t}(x, \cdot) \in \mathbb{R}\right)=1$ for every $x \in \mathbb{R}^{J}$ so $P\left(S_{t}(x, \cdot) \in \mathbb{R} \forall x \in \mathbb{Q}^{J}\right)=1$, where $\mathbb{Q}$ denotes the rational numbers. Since $S_{t}(\cdot, \omega)$ are convex, this implies $P\left(S_{t}(x, \omega) \in\right.$ $\left.\mathbb{R} \forall x \in \mathbb{R}^{J}\right)=1$.

${ }^{5}$ Here we use the fact that the integrability of $S$ implies that every selector of $\operatorname{dom} S_{t}^{*}$ is integrable.
} 
Remark 7 (Shadow prices) In unconstrained models with bid-ask spreads, the dual representation of the value function can be written

$\varphi(c)=\sup _{y \in \mathcal{M}^{\infty}}\left\{\langle c, y\rangle-E \sum_{t=0}^{T} v_{t}^{*}\left(y_{t}\right) \mid \exists s \in \mathcal{N}: s_{t}^{-} \leq s_{t} \leq s_{t}^{+}\right.$, ys is a martingale $\}$.

If the supremum is attained, the corresponding $s \in \mathcal{N}$ has the property that the optimum value of (ALM) is not affected if trading costs are reduced by replacing $S$ by the linear cost functions

$$
\tilde{S}_{t}(x, \omega)=s_{t}(\omega) \cdot x .
$$

Indeed, the corresponding value function $\tilde{\varphi}$ satisfies

$$
\begin{aligned}
\varphi(c) & \geq \tilde{\varphi}(c) \\
& \geq \sup _{y \in \mathcal{M}^{\infty}}\left\{\langle c, y\rangle-\tilde{\varphi}^{*}(y)\right\} \\
& =\sup _{y \in \mathcal{M}^{\infty}}\left\{\langle c, y\rangle-E \sum_{t=0}^{T} v_{t}^{*}\left(y_{t}\right) \mid \text { ys is a martingale }\right\} .
\end{aligned}
$$

where the first inequality holds because $S \geq \tilde{S}$ and the equality comes from Lemma 3. If $\varphi$ is proper and lower semicontinuous and if the supremum in its dual representation is attained by a $y \in \mathcal{M}^{\infty}$ and $s$, then the last supremum equals $\varphi(c)$. In [7], price processes s such that $\varphi(c)=\tilde{\varphi}(c)$ are called shadow prices. If the disutility functions $v_{t}$ are strictly increasing, then in the presence of a shadow price $s$, the optimal solution $x \in \mathcal{N}_{D}$ of (ALM), which exists under the assumptions of Theorem 2, must satisfy the complementarity conditions

$$
\Delta x_{t}>0 \Longrightarrow s_{t}=s_{t}^{+} \quad \text { and } \quad \Delta x_{t}<0 \Longrightarrow s_{t}=s_{t}^{-}
$$

since otherwise $x$ would achieve strictly lower trading costs and thus, a strictly lower objective value in the model with the linear cost functions $\tilde{S}_{t}$. Section 3 of $[7$,$] gives an example where shadow prices do not exist and thus, the supremum$ in the dual representation of $\varphi$ is not attained.

In the presence of a numeraire (see Example 1), the elements of $\mathcal{C}^{*}$ can be expressed in terms of probability measures.

Corollary 8 (Numeraire and martingale measures) Assume that $S$ is integrable and that

$$
S_{t}(x, \omega)=x^{0}+\tilde{S}_{t}(\tilde{x}, \omega) \quad \text { and } \quad D_{t}(\omega)=\mathbb{R} \times \tilde{D}_{t}(\omega),
$$

where $\tilde{S}$ is sublinear and $\tilde{D}$ is conical. Then ${ }^{6}$

$$
\mathcal{C}^{*}=\operatorname{pos}\left\{y \in \mathcal{M}_{+}^{\infty} \mid \exists Q \in \mathcal{P}: y_{t}=E_{t} \frac{d Q}{d P}\right\}
$$

where $\mathcal{P}=\left\{Q \ll P \mid \exists \tilde{s} \in \tilde{\mathcal{N}}: \tilde{s}_{t} \in \operatorname{dom} \tilde{S}_{t}^{*}, E_{t}^{Q} \Delta \tilde{s}_{t+1} \in \tilde{D}_{t}^{*} Q\right.$-a.s. $\}$ and $\tilde{\mathcal{N}}$ denotes the set of adapted $\mathbb{R}^{J \backslash\{0\}}$-valued processes.

\footnotetext{
${ }^{6}$ For a subset $C$ of a vector space, $\operatorname{pos} C:=\{\alpha x \mid \alpha \geq 0, x \in C\}$.
} 
Proof. We get

$$
\operatorname{dom} S_{t}=\{1\} \times \operatorname{dom} \tilde{S}_{t}^{*} \text { and } D_{t}^{*}=\{0\} \times \tilde{D}_{t}^{*}
$$

so, by Lemma $6, y \in \mathcal{C}^{*}$ iff $y$ is a nonnegative martingale and $E_{t}\left[\Delta\left(y_{t+1} \tilde{s}_{t+1}\right)\right] \in$ $\tilde{D}_{t}^{*}$ for some $\left(\mathcal{F}_{t}\right)_{t=0}^{T}$-adapted selector $\tilde{s}$ of $\operatorname{dom} \tilde{S}^{*}$ such that $y \tilde{s}$ is integrable. Clearly, $\mathcal{C}^{*}=\operatorname{pos}\left\{y \in \mathcal{C}^{*} \mid E y_{T}=1\right\}$. If $y \in \mathcal{C}^{*}$ with $E y_{T}=1$, then $y_{T}$ is the density of a probability measure $Q \ll P$ and, by [12, Proposition A.12],

$$
E_{t}^{Q}\left[\Delta \tilde{s}_{t+1}\right]=\frac{E_{t}\left[y_{T} \Delta \tilde{s}_{t+1}\right]}{E_{t} y_{T}}=\frac{E_{t}\left[\Delta\left(y_{t+1} \tilde{s}_{t+1}\right)\right]}{E_{t} y_{T}} \quad Q \text {-a.s. }
$$

Since $\tilde{D}_{t}^{*}$ is a cone, this implies $E_{t}^{Q}\left[\Delta \tilde{s}_{t+1}\right] \in \tilde{D}_{t}^{*} Q$-almost surely. Thus, $\{y \in$ $\left.\mathcal{C}^{*} \mid E y_{T}=1\right\} \subseteq\left\{y \in \mathcal{M}_{+}^{\infty} \mid \exists Q \in \mathcal{P}: y_{t}=E_{t} \frac{d Q}{d P}\right\}$. Conversely, if $y_{t}=$ $E_{t}[d Q / d P]$ for some $Q \in \mathcal{P}$, we get similarly that

$$
E_{t}\left[y_{T} \Delta \tilde{s}_{t+1}\right] \in \tilde{D}_{t}^{*} \quad Q \text {-a.s.. }
$$

Since $E_{t}\left[y_{T} \Delta \tilde{s}_{t+1}\right]=0 P$-almost surely on any $A \in \mathcal{F}_{t}$ with $Q(A)=0$ and since $0 \in \tilde{D}_{t}^{*}$, we get

$$
E_{t}\left[y_{T} \Delta \tilde{s}_{t+1}\right] \in \tilde{D}_{t}^{*} \quad P \text {-a.s. }
$$

and thus, $y \in \mathcal{C}^{*}$. We thus have

$$
\left\{y \in \mathcal{C}^{*} \mid E y_{T}=1\right\}=\left\{y \in \mathcal{M}_{+}^{\infty} \mid \exists Q \in \mathcal{P}: y_{t}=E_{t} \frac{d Q}{d P}\right\}
$$

which completes the proof.

In the classical linear model with $S_{t}(x, \omega)=s_{t}(\omega) \cdot x$ and $D_{t}=\mathbb{R}^{J}$, we have $\operatorname{dom} S_{t}^{*}=\left\{s_{t}\right\}$ and $D_{t}^{*}=\{0\}$ so the set $\mathcal{P}$ in Corollary 8 becomes the set of absolutely continuous martingale measures. In unconstrained models with bid-ask spreads, $\mathcal{P}$ is the set of absolutely continuous probability measures $Q$ such that there exists a $Q$-martingale $s$ with $s_{t}^{-} \leq s_{t} \leq s_{t}^{+}$for all $t$.

Example 9 In the setting of Corollary 8, the dual representation of $\varphi$ in Theorem 4 can be written as

$$
\begin{aligned}
\varphi(c) & =\sup _{y \in \mathcal{C}^{*}} E \sum_{t=0}^{T}\left[c_{t} y_{t}-v_{t}^{*}\left(y_{t}\right)\right] \\
& =\sup _{\lambda \geq 0} \sup _{Q \in \mathcal{P}} E \sum_{t=0}^{T}\left\{c_{t} \lambda E_{t} \frac{d Q}{d P}-\sum_{t=0}^{T} v_{t}^{*}\left(\lambda E_{t} \frac{d Q}{d P}\right)\right\} \\
& =\sup _{\lambda \geq 0} \sup _{Q \in \mathcal{P}}\left\{\lambda E^{Q} \sum_{t=0}^{T} c_{t}-E \sum_{t=0}^{T} v_{t}^{*}\left(\lambda E_{t} \frac{d Q}{d P}\right)\right\} .
\end{aligned}
$$

This is an illiquid discrete-time version of the dual problem of the optimal consumption problem from Karatzas and Žitković [21] which extends the duality 
framework of Kramkov and Schachermayer [23] by allowing consumption of wealth over time. When $v_{t}=\delta_{\mathbb{R}_{-}}$for $t<T$, we obtain a discrete-time version with illiquidity effects of the duality results in Owen and Žitković [29] and Biagini, Frittelli and Graselli [3]. In the exponential case

$$
v_{T}(c)=\frac{1}{\alpha}\left(e^{\alpha c}-1\right),
$$

we get $v_{T}^{*}(y)=(y \ln y-y+1) / \alpha$ and the supremum over $\lambda$ is attained at

$$
\lambda=\exp \left(E\left(\alpha \sum_{t=0}^{T} c_{t} y_{t}-y_{T} \ln y_{T}\right)\right) .
$$

This gives

$$
\begin{aligned}
\varphi(c) & =\sup _{Q \in \mathcal{P}}\left\{\frac{1}{\alpha} \exp \left[\alpha E^{Q} \sum_{t=0}^{T} c_{t}-H(Q \mid P)\right]-\frac{1}{\alpha}\right\} \\
& =\frac{1}{\alpha} \exp \left[\sup _{Q \in \mathcal{P}}\left\{\alpha E^{Q} \sum_{t=0}^{T} c_{t}-H(Q \mid P)\right\}\right]-\frac{1}{\alpha} .
\end{aligned}
$$

In the linear case $\tilde{S}_{t}(x)=\tilde{s}_{t} \cdot x$, this reduces to a discrete-time version of the duality framework of [9]; see also [20] and [1].

\section{Conclusions}

This paper studied optimal investment in the general conjugate duality framework of convex analysis. This has the benefit of allowing for various generalizations to the classical market models based on the theory of stochastic integration. In particular, the introduction of portfolio constraints and nonlinear illiquidity effects poses no particular problems compared to the classical model of perfectly liquid markets. One could also include dividend payments as proposed in [4]. Separation of dividend payments from "total returns" is important in the presence of transaction costs.

The optimization problem studied in this paper has important applications in accounting and in the valuation of swap contracts and other financial products with multiple payout dates. This has been studied in [34]. The related duality theory will be developed elsewhere.

\section{References}

[1] D. Becherer. Rational hedging and valuation of integrated risks under constant absolute risk aversion. Insurance Math. Econom., 33(1):1-28, 2003.

[2] S. Biagini and M. Frittelli. A unified framework for utility maximization problems: An orlicz spaces approach. The Annals of Applied Probability, 18(3):929-966, 2008. 
[3] S. Biagini, M. Frittelli, and M. Grasselli. Indifference price with general semimartingales. Mathematical Finance, 21(3):423-446, 2011.

[4] T. Bielecki, I. Cialenco, and R. Rodriguez. No-arbitrage pricing for dividend-paying securities in discrete-time markets with transaction costs. Technical report, 2012.

[5] U. Çetin and L. C. G. Rogers. Modelling liquidity effects in discrete time. Mathematical Finance, 17(1):15-29, 2007.

[6] J. Cvitanić and I. Karatzas. Convex duality in constrained portfolio optimization. Ann. Appl. Probab., 2(4):767-818, 1992.

[7] C. Czichowsky, J. Muhle-Karbe, and W. Schachermayer. Transaction costs, shadow prices, and connections to duality. preprint, 2012.

[8] R. C. Dalang, A. Morton, and W. Willinger. Equivalent martingale measures and no-arbitrage in stochastic securities market models. Stochastics Stochastics Rep., 29(2):185-201, 1990.

[9] F. Delbaen, P. Grandits, T. Rheinländer, D. Samperi, M. Schweizer, and C. Stricker. Exponential hedging and entropic penalties. Math. Finance, 12(2):99-123, 2002.

[10] F. Delbaen and W. Schachermayer. The Mathematics of Arbitrage. Springer Finance. Springer-Verlag, Berlin Heidelberg, 2006.

[11] M. A. H. Dempster, I. V. Evstigneev, and M. I. Taksar. Asset pricing and hedging in financial markets with transaction costs: An approach based on the Von Neumann-Gale model. Annals of Finance, 2(4):327-355, 2006.

[12] H. Föllmer and A. Schied. Stochastic finance. Walter de Gruyter \& Co., Berlin, extended edition, 2011. An introduction in discrete time.

[13] P. Guasoni. Optimal investment with transaction costs and without semimartingales. Ann. Appl. Probab., 12(4):1227-1246, 2002.

[14] P. Guasoni. Risk minimization under transaction costs. Finance Stoch., 6(1):91-113, 2002.

[15] J. M. Harrison and D. M. Kreps. Martingales and arbitrage in multiperiod securities markets. J. Econom. Theory, 20(3):381-408, 1979.

[16] J. M. Harrison and S. R. Pliska. Martingales and stochastic integrals in the theory of continuous trading. Stochastic Process. Appl., 11(3):215-260, 1981.

[17] E. Jouini and H. Kallal. Arbitrage in securities markets with short-sales constraints. Math. Finance, 5(3):197-232, 1995. 
[18] E. Jouini and H. Kallal. Martingales and arbitrage in securities markets with transaction costs. J. Econom. Theory, 66(1):178-197, 1995.

[19] Y. M. Kabanov and M. Safarian. Markets with transaction costs. Springer Finance. Springer-Verlag, Berlin, 2009. Mathematical theory.

[20] Y. M. Kabanov and Ch. Stricker. On the optimal portfolio for the exponential utility maximization: remarks to the six-author paper "Exponential hedging and entropic penalties" [Math. Finance 12 (2002), no. 2, 99-123; MR1891730 (2003b:91046)] by F. Delbaen, P. Grandits, T. Rheinländer, D. Samperi, M. Schweizer and C. Stricker. Math. Finance, 12(2):125-134, 2002 .

[21] I. Karatzas and G. Žitković. Optimal consumption from investment and random endowment in incomplete semimartingale markets. The Annals of Probability, 31(4):1821-1858, 2003.

[22] I. Klein and L. C. G. Rogers. Duality in optimal investment and consumption problems with market frictions. Math. Finance, 17(2):225-247, 2007.

[23] D. Kramkov and W. Schachermayer. The condition on the asymptotic elasticity of utility functions and optimal investment in incomplete markets. Annals of Applied Probability, 9(3):904-950, 1999.

[24] D. Kramkov and W. Schachermayer. Necessary and sufficient conditions in the problem of optimal investment in incomplete markets. Ann. Appl. Probab., 13(4):1504-1516, 2003.

[25] D. M. Kreps. Arbitrage and equilibrium in economies with infinitely many commodities. J. Math. Econom., 8(1):15-35, 1981.

[26] P. Malo and T. Pennanen. Reduced form modeling of limit order markets. Quantitative Finance, 12(7):1025-1036, 2012.

[27] J.-J. Moreau. Fonctionelles convexes. Séminaire sur les Equations aux Dérivées Partielles. Collège de France, Paris, 1967.

[28] C. Napp. The Dalang-Morton-Willinger theorem under cone constraints. $J$. Math. Econom., 39(1-2):111-126, 2003. Special issue on equilibrium with asymmetric information.

[29] M. P. Owen and G. Žitković. Optimal investment with an unbounded random endowment and utility-based pricing. Math. Finance, 19(1):129159, 2009.

[30] T. Pennanen. Arbitrage and deflators in illiquid markets. Finance and Stochastics, 15(1):57-83, 2011.

[31] T. Pennanen. Convex duality in stochastic optimization and mathematical finance. Mathematics of Operations Research, 36(2):340-362, 2011. 
[32] T. Pennanen. Dual representation of superhedging costs in illiquid markets. Mathematics and Financial Economics, 5:233-248, 2011.

[33] T. Pennanen. Superhedging in illiquid markets. Mathematical Finance, 21(3):519-540, 2011.

[34] T. Pennanen. Optimal investment and contingent claim valuation in illiquid markets. manuscript, 2013.

[35] T. Pennanen and A.-P. Perkkiö. Stochastic programs without duality gaps. Mathematical Programming, 136(1):91-110, 2012.

[36] M. Rásonyi and L. Stettner. On utility maximization in discrete-time financial market models. Ann. Appl. Probab., 15(2):1367-1395, 2005.

[37] R. T. Rockafellar. Convex analysis. Princeton Mathematical Series, No. 28. Princeton University Press, Princeton, N.J., 1970.

[38] R. T. Rockafellar. Conjugate duality and optimization. Society for Industrial and Applied Mathematics, Philadelphia, Pa., 1974.

[39] R. T. Rockafellar and R. J.-B. Wets. Variational analysis, volume 317 of Grundlehren der Mathematischen Wissenschaften [Fundamental Principles of Mathematical Sciences]. Springer-Verlag, Berlin, 1998.

[40] L. C. G. Rogers. Optimal Investment. Springer, Berlin, 2013.

[41] D. B. Rokhlin. An extended version of the Dalang-Morton-Willinger theorem under convex portfolio constraints. Theory Probab. Appl., 49(3):429443, 2005.

[42] W. Schachermayer. A Hilbert space proof of the fundamental theorem of asset pricing in finite discrete time. Insurance Math. Econom., 11(4):249257, 1992.

[43] W. Schachermayer. The fundamental theorem of asset pricing under proportional transaction costs in finite discrete time. Math. Finance, 14(1):1948, 2004. 